\title{
Motion characterization scheme to minimize motion artifacts in intravital microscopy
}

Sungon Lee

Gabriel Courties

Matthias Nahrendorf

Ralph Weissleder

Claudio Vinegoni 


\title{
Motion characterization scheme to minimize motion artifacts in intravital microscopy
}

\author{
Sungon Lee, ${ }^{\mathrm{a}}$ Gabriel Courties, ${ }^{\mathrm{b}}$ Matthias Nahrendorf, ${ }^{\mathrm{b}}$ Ralph Weissleder, ${ }^{\mathrm{b}}$ and Claudio Vinegoni ${ }^{\mathrm{b}, *}$ \\ aHanyang University, School of Electrical Engineering, Ansan, Republic of Korea \\ ${ }^{\mathrm{b}}$ Richard B. Simches Research Center, Center for Systems Biology, Massachusetts General Hospital and Harvard Medical School, \\ Boston, Massachusetts, United States
}

\begin{abstract}
Respiratory- and cardiac-induced motion artifacts pose a major challenge for in vivo optical imaging, limiting the temporal and spatial imaging resolution in fluorescence laser scanning microscopy. Here, we present an imaging platform developed for in vivo characterization of physiologically induced axial motion. The motion characterization system can be straightforwardly implemented on any conventional laser scanning microscope and can be used to evaluate the effectiveness of different motion stabilization schemes. This method is particularly useful to improve the design of novel tissue stabilizers and to facilitate stabilizer positioning in real time, therefore facilitating optimal tissue immobilization and minimizing motion induced artifacts. $\odot 2017$ Society of Photo-Optical Instrumentation Engineers (SPIE) [DOI: 10.1117/1.JBO.22.3.036005]
\end{abstract}

Keywords: motion artifact; intravital microscopy; motion compensation; in vivo imaging.

Paper 160455R received Jul. 1, 2016; accepted for publication Feb. 13, 2017; published online Mar. $2,2017$.

\section{Introduction}

Intravital fluorescence microscopy (IVM) offers high spatial and temporal resolution and enables multireporter visualization in live animals. As a result, IVM has emerged as one of the essential tools for obtaining cellular and molecular information under natural physiological conditions. Unfortunately, motion from various physiological processes, such as breathing and cardiovascular activity, continues to limit resolution and results in image artifacts.

While one would expect these artifacts to only be most pronounced in heart and lungs, they occur throughout the body, affecting every tissue at different temporal and spatial imaging scales. For these reasons, our understanding of various organ's natural physiology and function within the living body remains limited and, in some cases, it has almost exclusively relied on in vitro studies (e.g., Langendorff preparations for heart imaging).

To address this problem, several motion suppression techniques have been developed, but their use has been largely restricted to organs that move slowly over a small amplitude range. In particular, cardiac imaging has been difficult due to the organ's inherent rapid contraction and considerable motion displacement.

Currently, most acquisition strategies are based on active tracking devices or passive tissue stabilization, and specific tissue/organ stabilizers have been designed. ${ }^{1-10}$ In active motion compensation, the relative distance between the imaged organ and the microscope objective is kept constant over time. ${ }^{11-16}$ The objective is typically attached to a moving mechanism, such as a piezo actuator used in combination with a feedback loop based on high-speed optical imaging (e.g., CCD, optical coherence interferometry), ${ }^{11-12,14}$ contact-type displacement sensors, ${ }^{13}$ or image correlation, ${ }^{15,16}$ which is used to estimate the motion for tissue tracking. ${ }^{11-13}$ Images are then acquired

*Address all correspondence to: Claudio Vinegoni, E-mail: cvinegoni@mgh. harvard.edu providing acquisition sessions virtually free of any motion artifact. While these approaches are elegant, they are quite expensive, complex, and not straightforward to implement in commercial systems. Also, some approaches ${ }^{15}$ present limitations in terms of the amount of motion speed they can compensate and are more suited for slow drifts movements and not for physiologically induced motion activity. Passive stabilizers are therefore more commonly used, offering great improvement in stabilization. Specifically, they reduce the amount of tissue motion while at the same time, if properly designed and placed, they introduce reproducibility in the tissue displacement over multiple physiological cycles. This last feature is of crucial importance and through the use of retrospective gated or prospective triggered acquisition schemes, ${ }^{3,4,17-20}$ in combination with image processing, enables motion artifact-free image reconstructions. Typically, passive stabilizers rely on a compressive cover slip ${ }^{5-9}$ or suctioning devices ${ }^{2,4}$ to hold tissue in place, and different possible configurations and designs can be customized for the organ of interest. ${ }^{19,20}$ To compare stabilizers' effectiveness and to facilitate their developmental and design phase, it is therefore crucial to evaluate their performances not only mechanically and through simulations but also in an in vivo setting, optimizing them for maximum artifact removal and induced motion reproducibility. This is typically achieved through visual inspection during real-time image acquisition. The mouse position is rearranged, and the stabilizer's physical parameters, such as angle of positioning or point of contact, are adjusted until stabilized (or minimally perturbed) images are acquired.

In most cases, the compressing force or the suctioning pressure has to be optimally adjusted to reduce motion while avoiding tissue damage. Furthermore, excessive physical organ immobilization can potentially restrict blood flow and thus alter the natural physiology. Therefore, minimum physical immobilization and restriction in motion amplitude is desirable

1083-3668/2017/\$25.00 @ 2017 SPIE 
as long as temporal or spatiotemporal redundancy is present and can be identified within the acquired images. ${ }^{10}$

To rely only on visual inspection for optimizing imaging conditions can be challenging and time consuming. We have therefore developed a motion characterization system for axial displacement measurements to facilitate motion artifact-free image acquisition and reconstruction. The system is mounted on a dual slide nosepiece of a conventional microscope, which allows for easy switching between motion analysis and imaging microscopy (Fig. 1). While in-plane motion is not directly measured (this would require the presence of a CCD camera), its effect is usually of lesser importance. If present, (a)

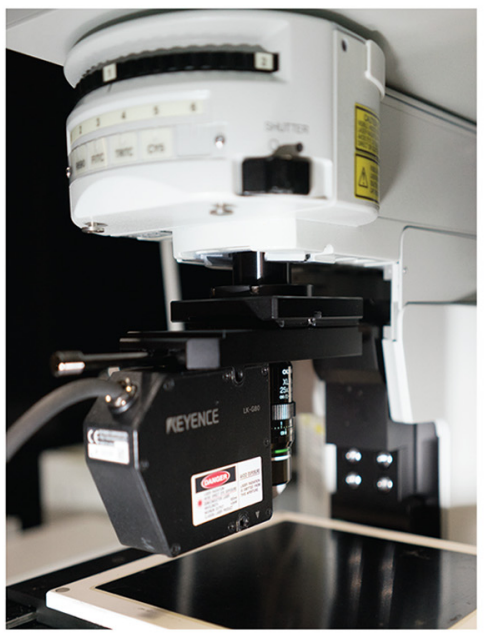

(b)

Motion Sensing

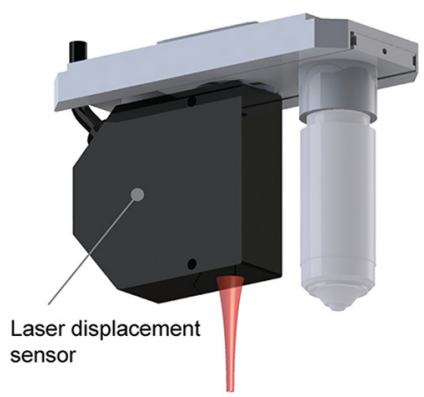

(d)

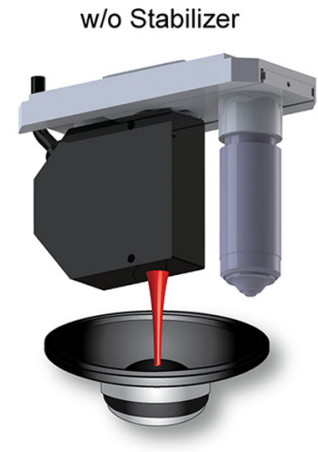

(g)

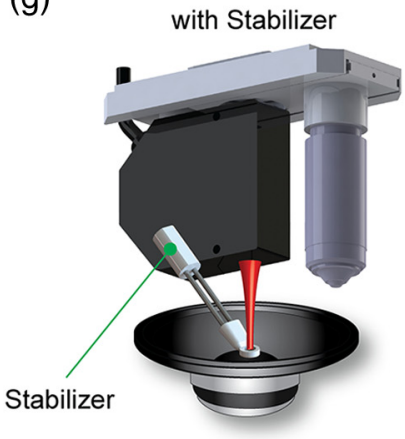

(e)

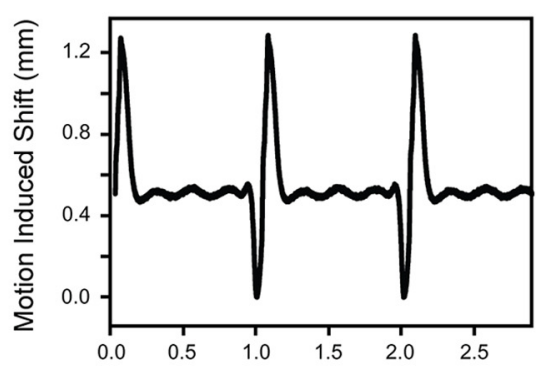

(h)

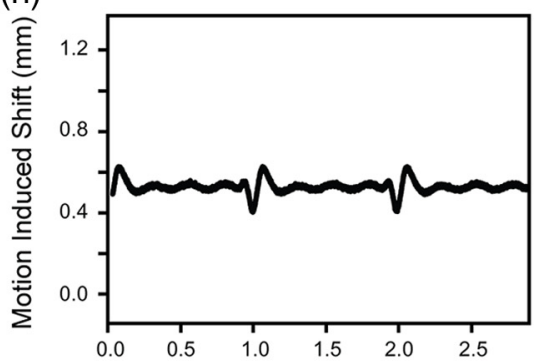

(c)

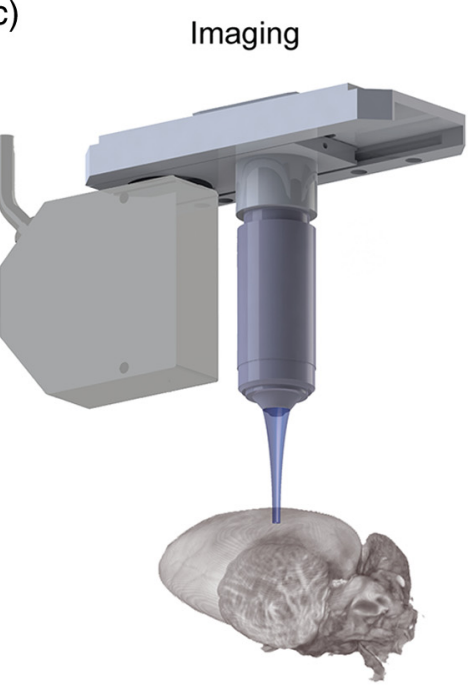

(f)

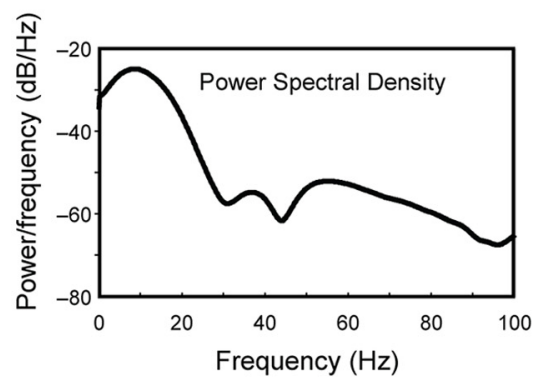

(i)

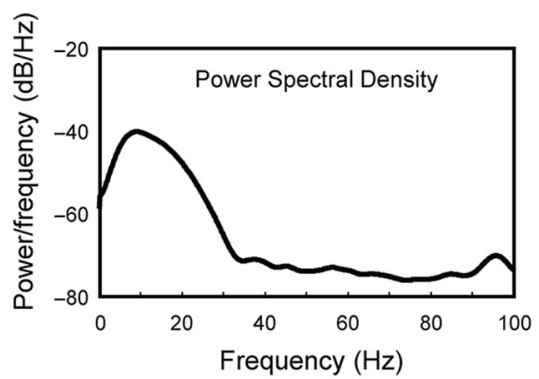

Fig. 1 Axial motion characterization system for in vivo imaging. (a) The setup is fully integrated within a commercial confocal/two photon microscope (FV1000, Olympus). A laser displacement sensor is mounted onto a dual slide nosepiece, collinear with the imaging objective. (b) During motion characterization, the sensor is positioned over the imaging organ, directly measuring the total displacement along the axial direction. (c) After mouse or stabilizer readjustment to reduce axial motion components and to improve reproducibility over the physiological cycles, the imaging objective is slid in position in the same spot, where the sensor was focused and image acquisition is performed. (d-i) For setup testing and proof-of-principle repetitive induced artificial motion was used. A sample fixed on top of a loudspeaker $(\mathrm{d}, \mathrm{g})$ is moved vertically with a synthetic waveform producing 1 and $5 \mathrm{~Hz}$ sinusoidal motion components, approximately equal to the mouse respiratory and cardiac frequencies. $(e, h)$ Amplitude measurements and $(f, i)$ frequency analysis are performed without $(d)$ and with $(g)$ the presence of a mechanical stabilizer. 
in-plane motion is usually connected to the axial component, and it can be easily removed via image processing.

\section{Experimental Methods}

The optical imaging set-up was based on a custom-modified Olympus FV1000-MPE (Olympus) laser scanning microscopy system adapted for small animal imaging and equipped with an upright BX61-WI microscope (Olympus) operating in both confocal and multiphoton mode. A large working distance water immersion objective (Olympus, XLPlan N W MP 25× 1.05 NA $2 \mathrm{~mm}$ WD) was used during imaging sessions. For sensing, a submicron-precision laser displacement sensor unit (Keyence LG-030) was mounted onto an objective holder sliding nosepiece, allowing to easily switch between the imaging objective and the sensor, without repositioning the imaged animal. The working principle of the sensor is based on simple triangulation to measure the distance from a surface. A laser beam is emitted and focused on the imaging sample, and the position of the reflection from the surface is recorded with a CCD sensing element. Because the imaging axis of the CCD is slightly tilted with respect to illumination, the position of the focused spot can be processed to find the absolute distance of the surface from the sensor itself. The imaging sensor offers a reproducibility of $0.05 \mu \mathrm{m}$, with a linearity of $\pm 0.05 \%$ on a measurement range of $\pm 5 \mathrm{~mm}$. The sampling rate was set to $5 \mathrm{KHz}$, with a sensing spot diameter of $\sim 200 \mu \mathrm{m}$. The laser displacement

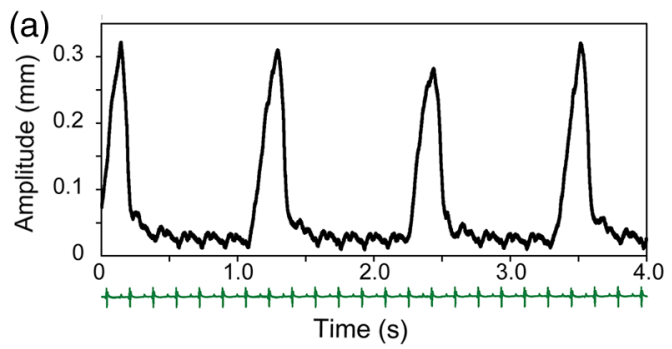

(b)

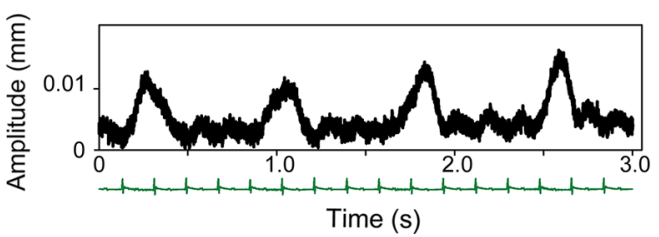

(c) Power Spectral Density Estimate
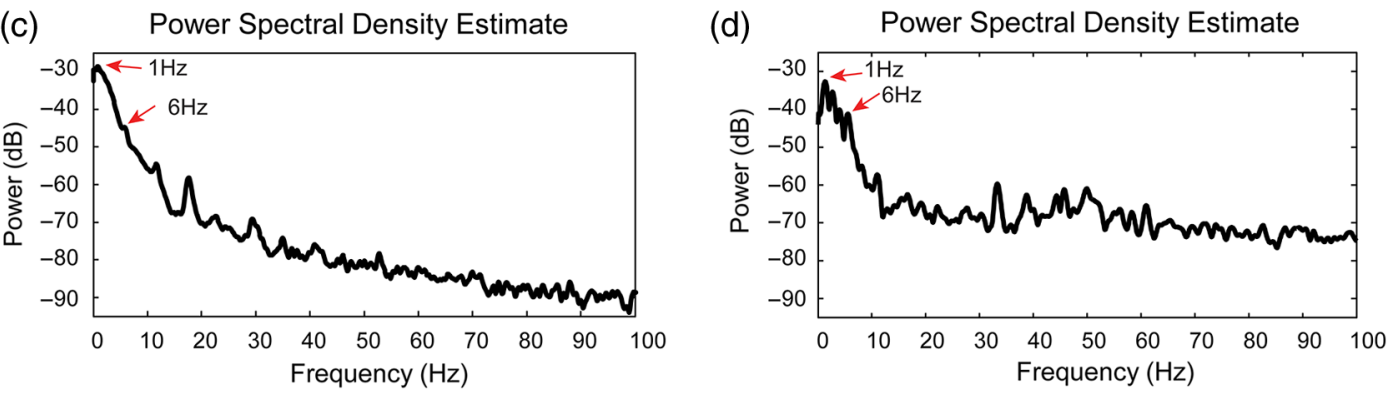

(e)
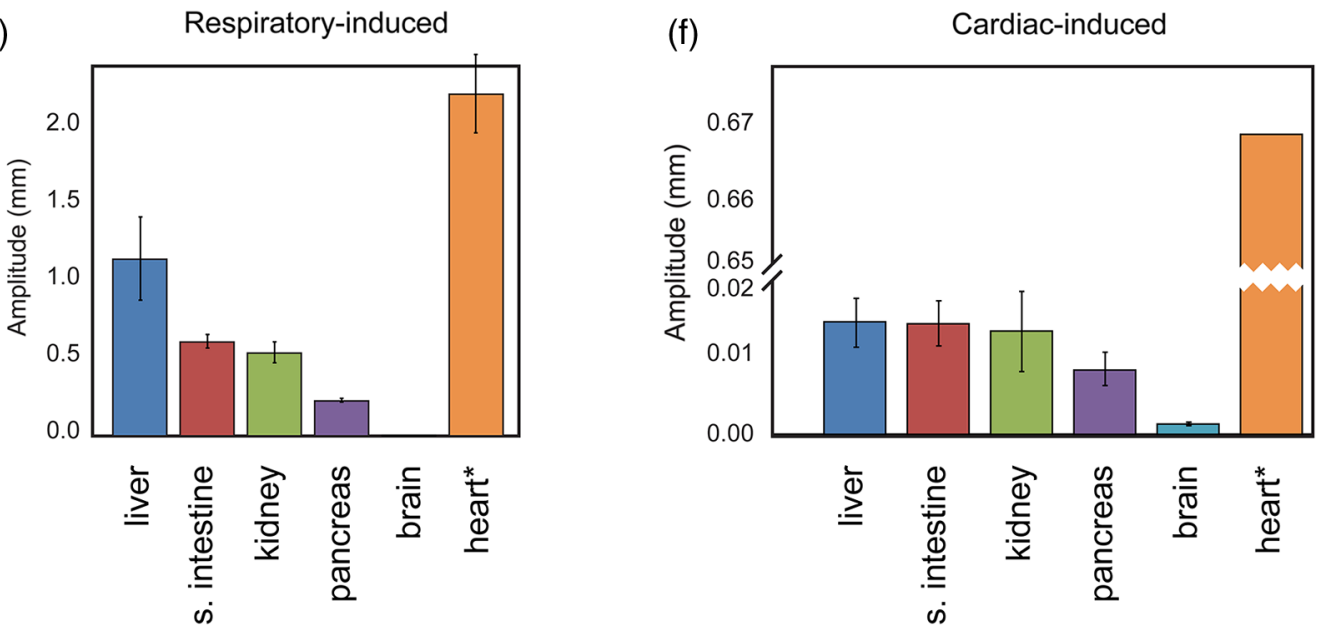

Fig. 2 In vivo motion characterization. (a) A typical example of tissue movement as measured by the custom-made motion characterization system. Two dominant repetitive motions are observed. The one with big amplitude is due to respiration and the small one due to cardiac activity. The ECG measurement in green color confirms that the small movement is caused by the heartbeat, and it is synchronized with motion. (b) A measurement of mouse brain movement during an imaging session. (c,d) Frequency analysis of (a) and (b), respectively, indicates major peaks at $\sim 1$ and $6 \mathrm{~Hz}$ corresponding to respiration and cardiac motion frequency. (e,f) Peak-to-peak displacements as measured at various organs and induced by (e) respiratory and $(f)$ cardiac activity during surgically open mouse imaging. Errors in (e) are calculated by measuring displacements over a 5 -s period, and averaging three times over continuous traces acquired for $1 \mathrm{~min}$. Errors in (f) are calculated by considering parts of the traces where respiratory motion is absent and averaging over multiple cardiac-induced peak-to-peak samples. ${ }^{*}$ indicates that an artificial animal ventilator was employed for heart motion characterization. Here, a calculated error of $40 \mu \mathrm{m}$ is present. 
sensor was positioned on the slider such that the sensing laser spot coincided approximately with the objective imaging focal point. To achieve this, the holder was designed such that the sensor could be translated along the $x$ and $y$ direction with the use of two sliders controlled by hexagonal screwdrivers. This allowed one to roughly adjust the planar position of the sensor's laser beam $(\sim 200 \mu \mathrm{m})$. Due to the large depth of field of the sensing unit (less than $300 \mu \mathrm{m}$ across $7 \mathrm{~mm}$ ), proper machining of the dual slide nosepiece guarantees that the imaging laser beam falls within the laser sensing volume. To test and prove the principle of the proposed setup, repetitive artificial motion was synthesized with a loudspeaker, as shown in Figs. 1(d)-1(i). An imaging sample was fixed on top of the speaker and moved vertically with a waveform mimicking mouse respiratory and cardiac movement at $1-$ and $5-\mathrm{Hz}$ frequency.

Movement along the $z$ direction was then measured to evaluate the degree of tissue stabilization. Amplitude measurements and frequency analyses were performed with and without a mechanical stabilizer.

During intravital imaging sessions, mice were anesthetized with $2 \%$ isoflurane and $21 / \mathrm{min}$ oxygen, and the body temperature of the mice was kept constant at $37^{\circ} \mathrm{C}$ during all procedures (surgery and imaging). For mice ventilation, an animal ventilator (Harvard Apparatus INSPIRA ASV 55-7058) was used. The ECG signal, recorded using three needle electrodes subcutaneously placed in the two front legs and the right hind leg, was filtered and amplified using a differential preamplifier (ADInstruments DP-301). Both ECG and ventilator traces were recorded using a data acquisition card (National Instruments, NI PCI-6229) $)^{19,20}$ and Labview software. Data and image processing were performed using MATLAB software (MATLAB 2014, Mathworks). For intravital imaging purposes C57BL/6, wild-type (Jackson Labs) mice were used as well as transgenic mouse strains, including mice-expressing fluorescent reporters of interest.

\section{Results}

\subsection{In Vivo Motion Characterization}

We next tested our setup during an in vivo mouse imaging session. All experimental animal protocols and procedures were approved by the Institutional Animal Care and Use Committee of the Massachusetts General Hospital, and they are in accordance with the NIH Guide for Care and Use of Laboratory Animals. Figure 2 shows typical examples of organs' vertical displacements as measured at the microscope imaging focal point, with the corresponding power spectral densities. The motion amplitudes were measured during in vivo lung $(a, c)$ and brain (b,d) imaging. The graph in Fig. 2(a) represents the displacement as recorded by the laser sensor, containing two distinct motion components. The large repetitive movement with a frequency of $\sim 1 \mathrm{~Hz}$ was caused by natural respiration while the small repetitive movement with a higher frequency around $\sim 6 \mathrm{~Hz}$ was due to cardiac activity. Figure 2(b) shows a measurement of brain movement in an open skull imaging session during which the mouse is stabilized with a stereotactic brain holder. Although very small, the movements induced by both spontaneous breathing and cardiac activity were observed. Correlation between simultaneously recorded ECG (green) and displacement data confirms that the few micron small, repetitive fluctuation was caused by the heartbeat. The peaks' frequencies of the power spectral density [Figs. 2(c) and 2(d)] show the presence of these two frequency components. Also, the power spectral density shows that the frequency component bigger than $20 \mathrm{~Hz}$ is very weak representing less than $-60 \mathrm{~dB}$ power.

We then proceeded to characterize how different organs move when surgically exposed. Using our motion characterization system, we first positioned our objective into an area of interest, and then slid the holder to bring the sensor in place. The recorded temporal traces were very similar to the ones shown in Fig. 2(a). The only major difference across organs was the magnitude of the peak-to-peak displacements. In Figs. 2(e)-2(f), we report values of motion amplitude as measured on mouse liver, small intestine, kidney, pancreas, brain, and heart. For each organ, both respiratory and cardiac induced peak-to-peak displacements are given. To note that data can vary among different mice but are indicative of the amount of motion present at different organs due to respiratory and cardiac physiological activities. These values can, in general, depend on different parameters, such as the mouse age, how the surgery has been performed, type of exteriorization or exposure of the imaged organ, amount of anesthesia administered, and mouse tolerance to the anesthesia itself.

We found that peak-to-peak displacements, on average, were $\sim 1.15 \mathrm{~mm}$ for the liver, the small intestine $0.61 \mathrm{~mm}$, the kidney $0.54 \mathrm{~mm}$, the pancreas $0.23 \mathrm{~mm}$, the brain $0.01 \mathrm{~mm}$, and the heart $2.22 \mathrm{~mm}$, respectively. Because heart imaging can be performed only with an open chest cavity, the total amplitude for this scenario was measured under assisted mechanical ventilation. As expected, respiratory-induced motion had the most significant impact on motion amplitude. The amount of total displacement due to cardiac activity is reported in Fig. 2(f), and it is $\sim 100$ times smaller than the displacement induced by respiration, except when imaging the heart itself, which presents the biggest peak-to-peak amplitude of about $0.67 \mathrm{~mm}$ on the average. Generally speaking, the motion amplitude of other organs, as affected by the heartbeat, was far less than that of the heart itself with $<0.02 \mathrm{~mm}$, which corresponds to only $3 \%$ of the peak-to-peak heart displacement. The heart instead showed the biggest peak-to-peak amplitude both by respiration and by cardiac activity, making it very difficult to stabilize during in vivo imaging sessions.

In addition to the displacement measurements, maximum velocities of organ movement were estimated from displacement measurements. To calculate the speed, a low pass filter, Butterworth filter fifth order having $40-\mathrm{Hz}$ cut-off frequency,

Table 1 Maximum velocity of various organs.

\begin{tabular}{lc} 
Organs & Maximum velocity $(\mathrm{mm} / \mathrm{s})$ \\
\hline Liver & 17.7 \\
Small intestine & 8.7 \\
Kidney & 4.4 \\
Pancreas & 5.1 \\
Brain & 0.2 \\
Heart (with ventilation) & 47.8 \\
Heart (no ventilation) & 19.9 \\
\hline
\end{tabular}


was applied to remove high frequency noise. Table 1 shows the estimated maximum velocities of organs from sample measurement data, with the heart having the highest maximum velocity among organs. Even intrinsic heart movement, measured with temporal suspension of mechanical ventilation, showed a very high velocity $(19.9 \mathrm{~mm} / \mathrm{s})$, as compared to other organs.

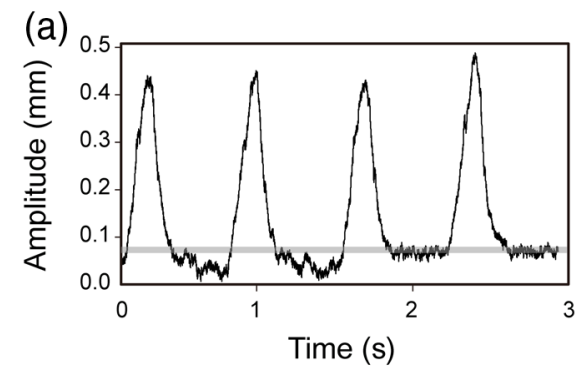

(c)

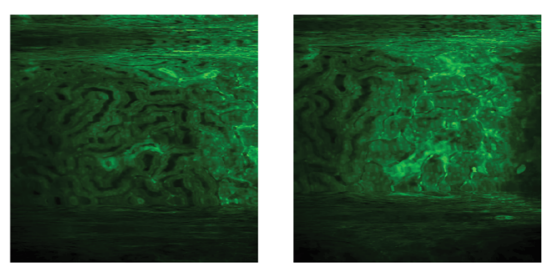

(f)

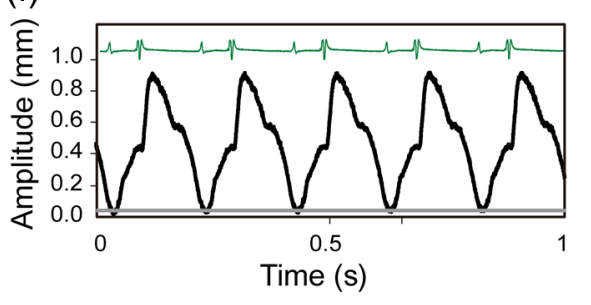

(h)

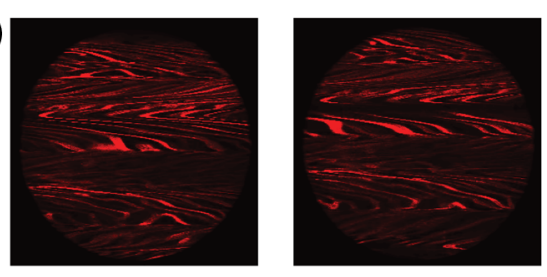

\subsection{Motion Stabilization and In Vivo Imaging}

Due to the large values of peak-to-peak motion amplitude, both respiratory- and cardiac-induced motion artifacts will impact image acquisitions. This is particularly true if images at the cellular level are acquired or if organs with large displacement

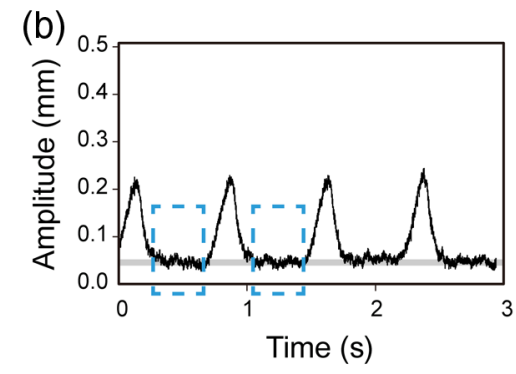

(d)

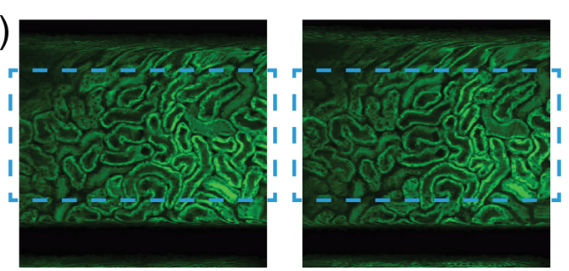

(e)

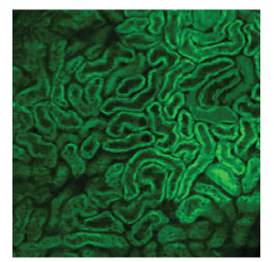

(g)

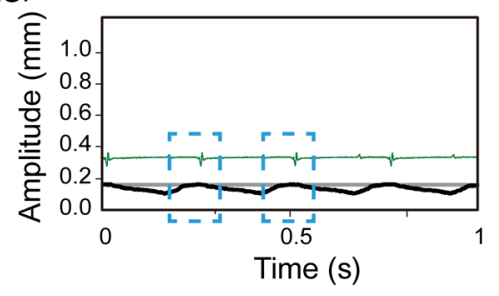

(i)
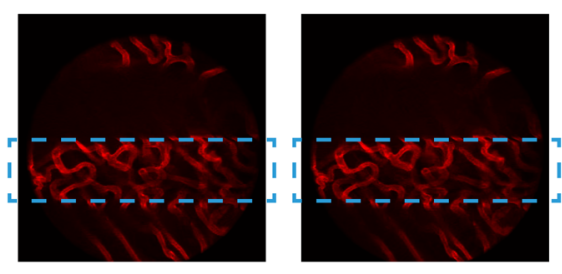

(j)

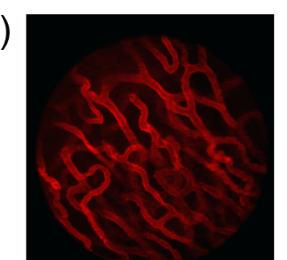

Fig. $3(a-e)$ Intravital imaging and motion detection of a kidney and $(f-j)$ the heart in a mouse. $(a, b)$ Traces representing the motion amplitude without and with the use of a mechanical stabilizer. (c, d) Representative sequential fluorescence images from two imaging acquisitions. (e) Example of an image reconstruction using stabilized images as obtained in (d). Fluorescence signal, here in green, is from the ubiquitin/green fluorescent protein expressing mice. (f, g) Traces representing the cardiac motion amplitude without and with the use of an adhesive stabilizer. (h, i) Representative sequential fluorescence images from two imaging acquisitions of the cardiac vasculature. (j) Example of an image reconstruction using stabilized images as obtained in (i). Here, the vasculature (in red color) was labeled with lectin. 
(such as lungs or the heart) are considered. Our imaging setup allows easy quantification of the total vertical range of motion and characterization of the extent of stabilization reached with a tissue stabilizer during imaging sessions. Before placing the stabilizer, it is important to reduce the free range of motion as much as possible to avoid applying excessive pressure onto the imaged tissue. For example, readjustment of the mouse position can highly impact the peak-to-peak amplitude. Such is the case when the mouse thoracic cage is firmly pressed against a heating plate or a mouse holder, and the respiratory motion transfers through the mouse cage to all other organs.

After this initial adjusting phase, the stabilizer (e.g., a simple pressure cover slip, a suctioning device or a positive pressure mechanical stabilizer) was set in place and tissue movement along the $z$ direction was measured to evaluate the degree of tissue stabilization necessary for the desired imaging resolution and objective magnification. Adjustments were then performed in the stabilizers' settings, such as the angle of incidence, the amount of positive or negative pressure, and relative position. Their impact was monitored in real time using the developed motion sensor, and when optimal conditions were reached, imaging sessions were initiated. There are two main factors to consider when trying to achieve motion stabilization. A first factor is the peak amplitude. Reduced peak amplitude leads to decreased imaging artifacts within a single image. The second factor is the reproducibility of movement. If quasiperiodic motion components are introduced, reproducibility of tissue position at certain time points during physiological cycles becomes predictable, allowing for assisted motion-synchronized scanning through prospective triggering or retrospective gating acquisition schemes. This improved reproducibility enables image reconstruction using image processing from multiple images as proposed and demonstrated earlier. ${ }^{3-5}$ This factor is far more important when the applied force/pressure cannot be increased to avoid the tissue damage (e.g., during heart imaging).

In Fig. 3, we show two different examples of motion characterization and imaging of mouse heart and kidney before and after tissue stabilization with accurate stabilizer adjustment. Specifically, we compared two different kinds of tissue stabilizers. A compressive motion stabilizer ${ }^{5}$ was used to reduce the movement of the mouse kidney. The position traces [Figs. 3(a) and 3(b)] acquired with the sensor show how the motion amplitude can be reduced with the presence of the tissue stabilizer, with the peak amplitude decreasing from $\sim 0.40$ to $0.15 \mathrm{~mm}$. With stronger pressure, this amplitude can be reduced further. However, as pointed out previously, ${ }^{5}$ it is more important to stabilize the position between each breath for postreconstruction than to reduce the peak amplitude. As shown in the figure, the vertical position between consecutive breaths becomes reproducible with the use of the tissue stabilizer, whereas it was occurring randomly without it. Here, the gray line indicates points in time with the same absolute vertical position. The images in Fig. 3(d) confirm the introduced reproducibility in the sequences acquired after stabilization, which can be further exploited for image processing. ${ }^{5}$ A complete image [Fig. 3(e)] can thus be reconstructed by combining a stabilized part from each image from a single acquisition into one. For this to occur, the image acquisition frequency should be adjusted to be slight off phase with the breathing frequency.

In Figs. 3(f)-3(j), a mouse heart was analyzed. The animal ventilator was stopped for a few seconds so to register only the intrinsic heart motion. Figure 3(f) shows the heart motion trace and the corresponding image sequence acquired without stabilizer [Fig. 3(h)], while in Fig. 3(g), motion is measured in the presence of a tissue bonding stabilizer. ${ }^{3}$ The sensor indicates that the heart moves almost $0.9 \mathrm{~mm}$ in its peak-to-peak displacement, and that these movements occur continuously even at diastole. After placing a custom-made stabilizer and after relative adjustments, the movement decreased to $<0.1 \mathrm{~mm}$ in its peak-to-peak amplitude. More importantly, a stable temporal window within the cardiac cycle (blue dashed rectangle) appeared at end diastole just before the QRS complex of the ECG. Furthermore, within this temporal window, the heart motion is reproducible between different heartbeats, as indicated by the gray line in Fig. 3(g). Images acquired within this time window (blue dashed boxes) show good reproducibility [Fig. 3(i)]. ${ }^{5}$

Similarly to before, assisted motion-synchronized scanning through retrospective gating acquisition allows an artifact-free image reconstruction [Fig. 3(j)].

\section{Conclusions}

In conclusion, we have developed an axial motion characterization system for intravital microscopy. We demonstrate measurement and analysis of various organs' movements. Conventionally, tissue stabilization for in vivo imaging can be only evaluated with "trial-and-error" procedures guided by visual inspection during real-time acquisition. Continuous readjustments are necessary until acquired images show minimal distortions, making the setup optimization process very time consuming. Furthermore, even if visual inspection can provide a first estimate of stabilization, it is far from accurate and images in most cases turn out to be insufficiently stabilized or not reproducible. This, in turn, can impede exploitation of triggered acquisitions for image processing reconstructions. The described motion characterization system enables to quantitate motion amplitude information in real time with great accuracy, facilitating optimal tissue stabilization conditions. It may further guide selection and design of the best-suited organ-specific stabilizer and adjustment parameters, which are of paramount importance for cellular high resolution in vivo imaging. While other schemes based on contact-type displacement sensors, ${ }^{13}$ or feedback loops based on high-speed CCD imaging, could be also adopted, ${ }^{11,12,14}$ the facile setup here illustrated can be implemented within a few hours in any conventional laser scanning microscopy system with minimal cost and effort.

\section{Disclosures}

No conflicts of interest, financial or otherwise, are declared by the authors.

\section{Acknowledgments}

We thank Rostic Gorbatov, Matt Sebas, John Dubach, for helpful discussions and assistance during surgical procedures. This project was funded by Grant Nos. P50CA086355, R01HL117829, R01EB010011, and R01HL122208. This work was also funded in part by the Korean Government (MSIP) under Grant No. 2015R1C1A1A01056013 and in part by Hanyang University (HY-2015-N). 


\section{References}

1. C. Vinegoni et al., "Advanced motion compensation methods for intravital optical microscopy," IEEE J. Sel. Top. Quantum Electron. 20(2), 83-91 (2014).

2. M. R. Looney et al., "Stabilized imaging of immune surveillance in the mouse lung," Nat. Methods 8(1), 91-96 (2011).

3. S. Lee et al., "Real-time in vivo imaging of the beating mouse heart at microscopic resolution," Nat. Commun. 3, 1054 (2012).

4. C. Vinegoni et al., "Motion compensation using a suctioning stabilizer for intravital microscopy," Intravital 1(2), 115-121 (2012).

5. S. Lee et al., "Improved intravital microscopy via synchronization of respiration and holder stabilization," J. Biomed. Opt. 17(9), 096018 (2012).

6. J. L. Li et al., "Intravital multiphoton imaging of immune responses in the mouse ear skin," Nat. Protocols 7(2), 221-234 (2012).

7. A. Holtmaat et al., "Long-term, high-resolution imaging in the mouse neocortex through a chronic cranial window," Nat. Protocols 4(8), 1128-1144 (2009).

8. K. W. Dunn et al., "Functional studies of the kidney of living animals using multicolor two-photon microscopy," Am. J. Physiol. Cell Physiol. 283(3), C905-C916 (2002).

9. H. A. Lehr et al., "Dorsal skinfold chamber technique for intravital microscopy in nude mice," Am. J. Pathol. 143(4), 1055-1062 (1993).

10. C. Vinegoni et al., "Sequential average segmented microscopy for high signal-to-noise ratio motion-artifact-free in vivo heart imaging," Biomed. Opt. Express 4(10), 2095-2106 (2013).

11. S. Lee et al., "Image stabilization for in vivo microscopy by high-speed visual feedback control," IEEE Trans. Rob. 24(1), 45-54 (2008).

12. S. Laffray et al., "Adaptive movement compensation for in vivo imaging of fast cellular dynamics within a moving tissue," PLoS One 6(5), e19928 (2011).

13. S. Lee et al., "In vivo microscope image stabilization through 3-D motion compensation using a contact-type sensor," in Proc. of IEEE/ RSJ Int. Conf. on Intelligent Robots and Systems, pp. 1192-1197 (2008).

14. Y. Huang et al., "Motion compensated fiber-optic confocal microscope based on a common-path optical coherence tomography distance sensor," Opt. Eng. 50(8), 083201 (2011).

15. M. Bakalar et al., "Three-dimensional motion tracking for high-resolution optical microscopy, in vivo," J. Microsc. 246(3), 237-247 (2012).

16. J. L. Schroeder et al., "Short communication: subcellular motion compensation for minimally invasive microscopy, in vivo: evidence for oxygen gradients in resting muscle," Circ. Res. 106(6), 1129-1133 (2010).

17. T. K. Foo et al., "Improved ejection fraction and flow velocity estimates with use of view sharing and uniform repetition time excitation with fast cardiac techniques," Radiology 195(2), 471-478 (1995).

18. J. M. Taylor et al., "Real-time optical gating for three-dimensional beating heart imaging," J. Biomed. Opt. 16(11), 116021 (2011).
19. C. Vinegoni et al., "Imaging the beating heart in the mouse using intravital microscopy techniques," Nat. Protocols 10, 1802-1819 (2015).

20. A.D Aguirre et al., "Intravital imaging of cardiac function at the singlecell level," Proc. Natl. Acad. Sci. 111, 11257-11262 (2014).

Sungon Lee received his $\mathrm{PhD}$ in 2008 and is currently an assistant professor in the School of Electrical Engineering, Hanyang University, Ansan, Republic of Korea. After completing his PhD degree in mechanoinformatics from the University of Tokyo, Japan, in 2008, he studied as a postdoctoral research fellow with the Center for Systems Biology at Massachusetts General Hospital, Harvard Medical School, United States, from 2010 to 2012 . His research interests include biomedical robotics, surgical robots, and medical image processing.

Gabriel Courties received his PhD in 2010. He is currently an instructor in radiology at Harvard Medical School and works at the Center for Systems Biology at Massachusetts General Hospital (MGH). He has published 28 original publications in peer-reviewed journals. His research interests involve the investigation of the interplay between immunology and cardiovascular diseases, including myocardial infarction, stroke, and atherosclerosis using intravital imaging, flow cytometry, and techniques for cell ablation or molecular deletion.

Matthias Nahrendorf is an associate professor at Harvard Medical School and director of the Mouse Imaging Program at the Center for Systems Biology at MGH. He completed his joint PhD and MD studies at the University of Heidelberg in Germany and his residency at the University of Würzburg. He joined Harvard Medical School in 2004. His laboratory at the MGH Center for Systems Biology focuses on cellular and molecular processes in atherosclerosis and myocardial infarction.

Ralph Weissleder is a professor at Harvard Medical School, director of the Center for Systems Biology at Massachusetts General Hospital (MGH), and attending clinician (interventional radiology) at $\mathrm{MGH}$. $\mathrm{He}$ is a member of the National Academies of Medicine, the American Academy of Arts and Sciences, and the German Academy of Science (Leopoldina). He has published over 800 publications in peer-reviewed journals and has authored several textbooks.

Claudio Vinegoni received his $\mathrm{PhD}$ in 2002 . He is currently an assistant professor at Harvard Medical School and works at the Center for Systems Biology at Massachusetts General Hospital (MGH). He completed his PhD at the University of Geneva in Switzerland. He has published over 90 original publications in peer-reviewed journals. His research activity involves the development of novel optical imaging instruments and techniques with applications in the clinical and biomedical arena. 